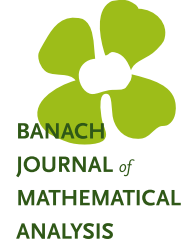

Banach J. Math. Anal. 11 (2017), no. 1, 1-20

http://dx.doi.org/10.1215/17358787-3721183

ISSN: $1735-8787$ (electronic)

http://projecteuclid.org/bjma

\title{
FRAMES AND REPRESENTING SYSTEMS IN FRÉCHET SPACES AND THEIR DUALS
}

\author{
J. BONET, ${ }^{1}$ C. FERNÁNDEZ,${ }^{2}$ A. GALBIS,${ }^{2}$ and J. M. RIBERA ${ }^{1}$ \\ Communicated by D. Bakić
}

\begin{abstract}
Frames and Bessel sequences in Fréchet spaces and their duals are defined and studied. Their relation with Schauder frames and representing systems is analyzed. The abstract results presented here, when applied to concrete spaces of analytic functions, give examples and consequences about sampling sets and Dirichlet series expansions.
\end{abstract}

\section{INTRODUCTION AND PRELIMINARIES}

The purpose of this article is twofold. On the one hand, we study $\Lambda$-Bessel sequences $\left(g_{i}\right)_{i} \subset E^{\prime}, \Lambda$-frames, and frames with respect to $\Lambda$ in the dual of a Hausdorff locally convex space $E$, in particular for Fréchet spaces and complete $(L B)$-spaces $E$ with a sequence space $\Lambda$. We investigate the relation of these concepts with representing systems in the sense of Korobeinik (see, e.g., Kadets and Korobeinik [13]) and with Schauder frames that were investigated by the authors in [8]. On the other hand, our article emphasizes the deep connection of frames for Fréchet and $(L B)$-spaces with sufficient and weakly sufficient sets for weighted Fréchet and $(L B)$-spaces of holomorphic functions. These concepts correspond to sampling sets in the case of Banach spaces of holomorphic functions. Our general results in Sections 2 and 3 permit us to obtain as a consequence examples and results in the literature in a unified way in Section 4, emphasizing their structural aspects.

Section 2 of our article is inspired by the work of Casazza, Christensen, and Stoeva [10] in the context of Banach spaces. Their characterizations of Banach

Copyright 2017 by the Tusi Mathematical Research Group.

Received Jul. 13, 2015; Accepted Feb. 12, 2016.

2010 Mathematics Subject Classification. Primary 46A04; Secondary 42C15, 46A13, 46E10.

Keywords. frames, representing systems, Fréchet spaces, $(L B)$-spaces, weakly sufficient sets. 
frames and frames with respect to a $B K$-sequence space gave us the proper hint to present here the right definitions in our more general setting (see Definition 2.1). The main result of this section is Proposition 2.8. A point of view different from ours concerning frames in Fréchet spaces was presented by Pilipovic and Stoeva in [24], [25]. Banach frames were introduced by Gröchenig. Shrinking and boundedly complete Schauder frames for Banach spaces were studied by Carando, Lassalle, and Schmidberg in [9]. Other precise references to work in this direction in the Banach space setting can be seen in [10]. Motivated by the applications to weakly sufficient sets for weighted $(L B)$-spaces of holomorphic functions, we present several abstract results about $\Lambda$-frames in complete $(L B)$-spaces that require a delicate analysis in Section 3. Our main result is Theorem 3.4. Finally, applications, results, and examples are collected in Section 4 concerning sufficient sets for weighted Fréchet spaces of holomorphic functions and weakly sufficient sets for weighted $(L B)$-spaces of holomorphic functions. We include here consequences related to the work of many authors (see [1]-[3], [7], [14], [17], [20], [21], [26], [29]).

Throughout this work, $E$ denotes a locally convex Hausdorff linear topological space (LCS), and CS $(E)$ is the system of continuous seminorms describing the topology of $E$. Sometimes additional assumptions on $E$ are added. The symbol $E^{\prime}$ stands for the topological dual of $E$ and $\sigma\left(E^{\prime}, E\right)$ for the weak* topology on $E^{\prime}$. We set $E_{\beta}^{\prime}$ for the dual $E^{\prime}$ endowed with the topology $\beta\left(E^{\prime}, E\right)$ of uniform convergence on the bounded sets of $E$. We will refer to $E_{\beta}^{\prime}$ as the strong dual of $E$. The Mackey topology $\mu\left(E^{\prime}, E\right)$ is the topology on $E^{\prime}$ of the uniform convergence on the absolutely convex and weakly compact sets of $E$. We refer to [12] for LCS. If $T: E \rightarrow F$ is a continuous linear operator, then its transpose is denoted by $T^{\prime}: F^{\prime} \rightarrow E^{\prime}$, and it is defined by $T^{\prime}(v)(x):=v(T(x)), x \in E, v \in F^{\prime}$. We recall that a Fréchet space is a complete meterizable LCS. An $(L B)$-space is an LCS that can be represented as an injective inductive limit of a sequence $\left(E_{n}\right)_{n}$ of Banach spaces. In most of the results we need the assumption that the LCS is barreled. The reason is that the Banach-Steinhaus theorem holds for barreled LCS. Every Fréchet space and every $(L B)$-space is barreled. We refer the reader to [12] and [23] for more information about barreled spaces.

As usual, $\omega$ denotes the countable product $\mathbb{K}^{\mathbb{N}}$ of copies of the scalar field, endowed with the product topology, and $\varphi$ stands for the space of sequences with finite support. A sequence space $\Lambda$ is an LCS which contains $\varphi$ and is continuously included in $\omega$. Note that $\Lambda$ is said to be solid if $x=\left(x_{i}\right)_{i} \in \Lambda$, and $\left|y_{i}\right| \leq\left|x_{i}\right|$ for all $i \in \mathbb{N}$ implies that $y=\left(y_{i}\right)_{i} \in \Lambda$.

Definition 1.1. Given a sequence space $\Lambda$, its $\beta$-dual space is defined as

$$
\Lambda^{\beta}:=\left\{\left(y_{i}\right)_{i} \in \omega: \sum_{i=1}^{\infty} x_{i} y_{i} \text { converges for every }\left(x_{i}\right)_{i} \in \Lambda\right\} .
$$

Clearly, $\left(\Lambda, \Lambda^{\beta}\right)$ is a dual pair. Under additional assumptions, we also have the relation given in the next essentially known lemma.

Lemma 1.2. Let $\Lambda$ be a barreled sequence $L C S$ for which the canonical unit vectors $\left(e_{i}\right)_{i}$ form a Schauder basis. Then its topological dual $\Lambda^{\prime}$ can be algebraically 
identified with its $\beta$-dual $\Lambda^{\beta}$, and the canonical unit vectors $\left(e_{i}\right)_{i}$ are a basis for $\left(\Lambda^{\beta}, \mu\left(\Lambda^{\beta}, \Lambda\right)\right)$. Moreover, if we consider on $\Lambda^{\beta}$ the system of seminorms given by

$$
p_{B}\left(\left(y_{i}\right)_{i}\right):=\sup _{x \in B}\left|\sum_{i} x_{i} y_{i}\right|,
$$

where $B$ runs on the bounded subsets of $\Lambda$, then $\left(\Lambda^{\beta},\left(p_{B}\right)_{B}\right)$ is topologically isomorphic to $\left(\Lambda^{\prime}, \beta\left(\Lambda^{\prime}, \Lambda\right)\right)$.

Proof. The map

$$
\psi: \Lambda^{\prime} \rightarrow \Lambda^{\beta}, \quad h \rightarrow\left(h\left(e_{i}\right)\right)_{i}
$$

is a linear bijection. In fact, for every $h \in \Lambda^{\prime}$ and $x=\left(x_{i}\right)_{i} \in \Lambda$, we have $h(x)=\sum_{i} x_{i} h\left(e_{i}\right)$, which implies that $\psi$ is well defined and obviously linear and injective. The barreledness of $\Lambda$ and the Banach-Steinhaus theorem give the surjectivity.

If $K \subset \Lambda$ is $\sigma\left(\Lambda, \Lambda^{\beta}\right)$-compact, then, given $y \in \Lambda^{\beta}$ and $\varepsilon>0$, there is $n_{0}$ such that, for $n \geq n_{0}$,

$$
\left|\sum_{i=n}^{\infty} y_{i} x_{i}\right|<\varepsilon
$$

for all $x \in K$ from where $y=\sum_{i} y_{i} e_{i}$ in the Mackey topology $\mu\left(\Lambda^{\beta}, \Lambda\right)$.

As for each bounded subset $B$ of $\Lambda$, we have

$$
p_{B}\left(\left(h\left(e_{i}\right)\right)_{i}\right)=\sup _{x \in B}|h(x)|,
$$

and the topological identity follows.

From now on, if the sequence space $\Lambda$ satisfies the assumption in Lemma 1.2, then we identify $\Lambda^{\prime}$ with $\Lambda^{\beta}$ and use always $\Lambda^{\prime}$.

\section{General Results}

Definition 2.1. Let $E$ be an LCS, and let $\Lambda$ be a sequence space.

(1) $\left(g_{i}\right)_{i} \subset E^{\prime}$ is called a $\Lambda$-Bessel sequence for $E$ if the analysis operator

$$
\begin{aligned}
U_{\left(g_{i}\right)_{i}}: E & \longrightarrow \Lambda, \\
x & \longmapsto\left(g_{i}(x)\right)_{i}
\end{aligned}
$$

is well defined and continuous.

(2) $\left(g_{i}\right)_{i} \subset E^{\prime}$ is called a $\Lambda$-frame for $E$ if the analysis operator $U=U_{\left(g_{i}\right)_{i}}$ is an isomorphism into its image. If, in addition, the range $U(E)$ of the analysis operator is complemented in $\Lambda$, then $\left(g_{i}\right)_{i}$ is said to be a frame for $E$ with respect to $\Lambda$. In this case there exists $S: \Lambda \rightarrow E$ such that $S \circ U=\left.\mathrm{id}\right|_{E}$.

For simplicity, when $\left(g_{i}\right)_{i}$ is clear from the context, the analysis operator will be denoted by $U$. Also, we can write $\Lambda$-Bessel sequence or $\Lambda$-frame instead of $\Lambda$-Bessel sequence for $E$ or $\Lambda$-frame for $E$.

Definition 2.1 is motivated by Definitions 1.2 and 1.3 in [10]. More precisely, we had in mind Theorems 2.1 and 2.4 in [10] that give the right idea on how 
to extend the definitions to the locally convex setting. $\Lambda$-frames and frames with respect to $\Lambda$ are called pre-frames and frames in [4].

Clearly, given an LCS $E$, each sequence $\left(g_{i}\right)_{i} \subset E^{\prime}$ is an $\omega$-Bessel sequence. On the other hand, if $\Lambda$ is a Hilbert space and $E$ is complete, then each $\Lambda$-frame is a frame with respect to $\Lambda$. Obviously, an LCS space has a $\Lambda$-frame if and only if it is isomorphic to a subspace of $\Lambda$, and it has a frame with respect to $\Lambda$ if and only if it is isomorphic to a complemented subspace of $\Lambda$. Therefore, the property of having a $\Lambda$-frame is inherited by subspaces, whereas having a frame with respect to $\Lambda$ is inherited by complemented subspaces.

Remark 2.2. Let $E$ be an LCS, let $\Lambda_{1}, \Lambda_{2}$ be sequence spaces, let $\left(g_{i}\right)_{i} \subset E^{\prime}$ be a $\Lambda_{1}$-Bessel sequence, and let $\left(h_{i}\right)_{i} \subset E^{\prime}$ be a $\Lambda_{2}$-frame. We define $\left(f_{k}\right)_{k} \subset E^{\prime}$ as $f_{k}=g_{i}$ for $k=2 i-1$ and $f_{k}=h_{i}$ when $k=2 i$. Consider the sequence space

$$
\Lambda:=\left\{\left(\alpha_{k}\right)_{k}:\left(\alpha_{2 k-1}\right)_{k} \in \Lambda_{1}, \text { and }\left(\alpha_{2 k}\right)_{k} \in \Lambda_{2}\right\}
$$

with the topology given by the seminorms

$$
\|\alpha\|_{p, q}:=p\left(\left(\alpha_{2 k-1}\right)_{k}\right)+q\left(\left(\alpha_{2 k}\right)_{k}\right), \quad \text { where } p \in \operatorname{CS}\left(\Lambda_{1}\right), q \in \operatorname{CS}\left(\Lambda_{2}\right) .
$$

Then $\left(f_{k}\right)_{k}$ is a $\Lambda$-frame for $E$. In the case that $\Lambda_{1}=\Lambda_{2}$ is one of the spaces $c_{0}$ or $\ell_{p}$, then $\Lambda=\Lambda_{1}=\Lambda_{2}$.

Let $E$ be an LCS, let $\left(x_{i}\right)_{i} \subset E$, and let $\left(x_{i}^{\prime}\right)_{i} \subset E^{\prime}$. We recall that $\left(\left(x_{i}^{\prime}\right)_{i},\left(x_{i}\right)_{i}\right)$ is said to be a Schauder frame for $E$ if

$$
x=\sum_{i=1}^{\infty} x_{i}^{\prime}(x) x_{i} \quad \text { for all } x \in E,
$$

the series converging in $E$. A Schauder frame for $E$ with the additional property that $\left(\left(x_{i}\right)_{i},\left(x_{i}^{\prime}\right)_{i}\right)$ is a Schauder frame for $E_{\beta}^{\prime}$ is called a shrinking Schauder frame (see [8]). The associated sequence space is

$$
\Lambda:=\left\{\alpha=\left(\alpha_{i}\right)_{i} \in \omega: \sum_{i=1}^{\infty} \alpha_{i} x_{i} \text { is convergent in } E\right\} .
$$

Endowed with the system of seminorms

$$
\mathcal{Q}:=\left\{q_{p}\left(\left(\alpha_{i}\right)_{i}\right):=\sup _{n} p\left(\sum_{i=1}^{n} \alpha_{i} x_{i}\right), \text { for all } p \in \operatorname{CS}(E)\right\},
$$

$\Lambda$ is a sequence space, and the canonical unit vectors form a Schauder basis.

There is a close connection between $\Lambda$-frames and Schauder frames.

Proposition 2.3. (1) Let $\left(\left(x_{i}^{\prime}\right)_{i},\left(x_{i}\right)_{i}\right)$ be a Schauder frame for a barreled and complete LCS E, and let $\Lambda$ be the associated sequence space. Then $\left(x_{i}^{\prime}\right)_{i} \subset$ $E^{\prime}$ is a frame for $E$ with respect to $\Lambda$. Moreover, if $\Lambda$ is barreled, then $\left(x_{i}\right)_{i} \subset E$ is a frame for $E^{\prime}$ with respect to $\Lambda^{\prime}$.

(2) If $\left(x_{i}^{\prime}\right)_{i} \subset E^{\prime}$ is a frame for $E$ with respect to a sequence space $\Lambda$, and if $\Lambda$ has a Schauder frame, then $E$ also admits a Schauder frame. 
Proof. (1) According to the proof of [8, Theorem 1.4], the operators $U: E \rightarrow \Lambda$ and $S: \Lambda \rightarrow E$ given by $U(x):=\left(x_{i}^{\prime}(x)\right)_{i}$ and $S\left(\left(\alpha_{i}\right)_{i}\right):=\sum_{i=1}^{\infty} \alpha_{i} x_{i}$, respectively, are continuous and $S \circ U=\mathrm{id}_{E}$. Consequently, $\left(x_{i}^{\prime}\right)_{i}$ is a frame for $E$ with respect to $\Lambda$. Under the additional assumption that $\Lambda$ is barreled, we have that $\Lambda^{\prime}=\Lambda^{\beta}$ is a sequence space. Moreover, $S^{\prime}\left(x^{\prime}\right):=\left(x^{\prime}\left(x_{i}\right)\right)_{i}$ for each $x^{\prime} \in E^{\prime}$, and from $U^{\prime} \circ S^{\prime}=\operatorname{id}_{E^{\prime}}$ we conclude that $\left(x_{i}\right)_{i}$ is a frame for $E^{\prime}$ with respect to $\Lambda^{\prime}$.

Statement (2) follows from the fact that having a Schauder frame is inherited by complemented subspaces.

The barreledness of the sequence space $\Lambda$ naturally associated to a Schauder frame follows, for instance, if $E$ is a Fréchet space. Observe that the dual space $E^{\prime}$ need not be separable, in which case neither need $\Lambda^{\prime}$ be separable.

The following two concepts were introduced by Korobeinik (see, for instance, $[15])$.

Definition 2.4. A representing system in an LCS $E$ is a sequence $\left(x_{i}\right)_{i}$ in $E$ such that each $x \in E$ admits a representation

$$
x=\sum_{i} c_{i} x_{i}
$$

the series converging in $E$.

The coefficients in the representation need not be unique; that is, one can have

$$
0=\sum_{i} d_{i} x_{i}
$$

for a nonzero sequence $\left(d_{i}\right)_{i}$. Moreover, we do not assume that it is possible to find a representation of this type with coefficients depending continuously on the vectors.

Clearly, each topological basis is a representing system. Given a Schauder frame $\left(\left(x_{i}^{\prime}\right)_{i},\left(x_{i}\right)_{i}\right)$, the sequence $\left(x_{i}\right)_{i}$ is a representing system. However, there are representing systems that are not a basis or coming from a Schauder frame. In fact, each separable Fréchet space has a representing system (see [15] and [13, Theorem 1]), but only those separable Fréchet spaces with the bounded approximation property admit a Schauder frame (see [8, Corollary 1.5]).

Definition 2.5. A $\Lambda$-representing system in a LCS $E$ is a sequence $\left(x_{i}\right)_{i}$ in $E$ such that each $x \in E$ admits a representation $x=\sum_{i} c_{i} x_{i}$ with $\left(c_{i}\right)_{i} \in \Lambda$.

Proposition 2.6. Let $E$ be a barreled $L C S$, and let $\Lambda$ be a barreled sequence LCS for which the canonical unit vectors $\left(e_{i}\right)_{i}$ form a Schauder basis. Then we have the following.

(1) (a) $\left(g_{i}\right)_{i} \subset E^{\prime}$ is a $\Lambda$-Bessel sequence if and only if the operator

$$
T:\left(\Lambda^{\prime}, \mu\left(\Lambda^{\prime}, \Lambda\right)\right) \rightarrow\left(E^{\prime}, \mu\left(E^{\prime}, E\right)\right), \quad\left(d_{i}\right)_{i} \mapsto \sum_{i=1}^{\infty} d_{i} g_{i}
$$

is well defined and continuous. 
(b) $\left(g_{i}\right)_{i} \subset E^{\prime}$ is $\Lambda^{\prime}$-Bessel for $E$ if and only if the operator

$$
T: \Lambda \rightarrow\left(E^{\prime}, \beta\left(E^{\prime}, E\right)\right), \quad\left(d_{i}\right)_{i} \mapsto \sum_{i=1}^{\infty} d_{i} g_{i}
$$

is well defined and continuous.

(2) If $\left(g_{i}\right)_{i} \subset E^{\prime}$ is a $\Lambda$-frame for $E$, then $\left(g_{i}\right)_{i}$ is a $\Lambda^{\prime}$-representing system for $\left(E^{\prime}, \mu\left(E^{\prime}, E\right)\right)$. Moreover, if $E$ is reflexive, then $\left(g_{i}\right)_{i}$ is a $\Lambda^{\prime}$-representing system for $\left(E^{\prime}, \beta\left(E^{\prime}, E\right)\right)$.

(3) If $\left(g_{i}\right)_{i} \subset E^{\prime}$ is a $\Lambda$-Bessel sequence which is also a $\Lambda^{\prime}$-representing system for $\left(E^{\prime}, \mu\left(E^{\prime}, E\right)\right)$, then $\left(g_{i}\right)_{i}$ is a $\left(\Lambda, \sigma\left(\Lambda, \Lambda^{\prime}\right)\right)$-frame for $\left(E, \sigma\left(E, E^{\prime}\right)\right)$.

(4) If in addition $E$ and $\Lambda$ are Fréchet spaces, then $\left(g_{i}\right)_{i} \subset E^{\prime}$ is a $\Lambda$-frame for $E$ if and only if $\left(g_{i}\right)_{i}$ is $\Lambda$-Bessel and a $\Lambda^{\prime}$-representing system for $\left(E^{\prime}, \mu\left(E^{\prime}, E\right)\right)$.

Proof. (1)(a) Let us assume that $\left(g_{i}\right)_{i}$ is a $\Lambda$-Bessel sequence and consider $T=U^{\prime}$ the transpose map of the analysis operator $U: E \rightarrow \Lambda, U(x)=\left(g_{i}(x)\right)_{i}$. Then $T:\left(\Lambda^{\prime}, \mu\left(\Lambda^{\prime}, \Lambda\right)\right) \rightarrow\left(E^{\prime}, \mu\left(E^{\prime}, E\right)\right)$ is continuous and $T\left(e_{i}\right)=g_{i}$. As the canonical unit vectors are a basis for $\left(\Lambda^{\prime}, \mu\left(\Lambda^{\prime}, \Lambda\right)\right)$, we conclude that $T\left(\left(d_{i}\right)_{i}\right)=\sum_{i=1}^{\infty} d_{i} g_{i}$. Conversely, if $T$ is a well-defined and continuous map, then its transpose $T^{\prime}$ : $E \rightarrow \Lambda$ is also continuous, which means that $\left(g_{i}\right)_{i}$ is a $\Lambda$-Bessel sequence. (1)(b) is proved similarly considering that the dual $\left(\Lambda^{\prime}, \beta\left(\Lambda^{\prime}, \Lambda\right)\right)$ is a sequence space.

(2) If $\left(g_{i}\right)_{i}$ is a $\Lambda$-frame, then $U$ is a topological isomorphism into its image; hence $T=U^{\prime}$ is surjective. In particular, $\left(g_{i}\right)_{i}$ is a $\Lambda^{\prime}$-representing system in $\left(E^{\prime}, \mu\left(E^{\prime}, E\right)\right)$.

(3) From (1), the map $T:\left(\Lambda^{\prime}, \mu\left(\Lambda^{\prime}, \Lambda\right)\right) \rightarrow\left(E^{\prime}, \mu\left(E^{\prime}, E\right)\right),\left(d_{i}\right)_{i} \mapsto \sum_{i=1}^{\infty} d_{i} g_{i}$, is well defined, continuous, and surjective. Consequently, $T^{\prime}:\left(E, \sigma\left(E, E^{\prime}\right)\right) \rightarrow$ $\left(\Lambda, \sigma\left(\Lambda, \Lambda^{\prime}\right)\right)$ is an isomorphism into its image [12, Proposition 9.6.1]; hence $\left(g_{i}\right)_{i}$ is a $\left(\Lambda, \sigma\left(\Lambda, \Lambda^{\prime}\right)\right)$-frame for $\left(E, \sigma\left(E, E^{\prime}\right)\right)$.

(4) Necessity follows from (2) and sufficiency follows from the closed-range theorem [12, Theorem 9.6.3] and (3).

Relevant consequences of Proposition 2.6 for spaces of analytic functions are given later in Theorem 4.4, which is due to Abanin and Khoi [2] and Corollary 4.10.

The next result is the extension in our context of [10, Corollary 3.3].

Proposition 2.7. Let $E$ be a reflexive space, and let $\Lambda$ be a reflexive sequence space for which the canonical unit vectors $\left(e_{i}\right)_{i}$ form a Schauder basis. If either

(1) $E$ and $\Lambda$ are Fréchet spaces

or

(2) $E$ is the strong dual of a Fréchet Montel space and $\Lambda$ is an $(L B)$-space, then $\left(g_{i}\right)_{i} \subset E^{\prime}$ is a $\Lambda$-frame for $E$ if and only if $\left(g_{i}\right)_{i}$ is $\Lambda$-Bessel and a $\Lambda^{\prime}$-representing system for $\left(E^{\prime}, \beta\left(E^{\prime}, E\right)\right)$.

Proof. The case (1) is Proposition 2.6(4). Only the sufficiency in case (2) has to be proved. Let us assume that $\left(g_{i}\right)_{i}$ is $\Lambda$-Bessel and a $\Lambda^{\prime}$-representing system for $\left(E^{\prime}, \beta\left(E^{\prime}, E\right)\right)$ and consider the continuous map $U: E \rightarrow \Lambda, U(x)=\left(g_{i}(x)\right)_{i}$. 
Then $T=U^{\prime}: \Lambda^{\prime} \rightarrow E^{\prime}$ is a well-defined, continuous and surjective map. Since $E^{\prime}$ is a Fréchet Montel space, the map $T$ lifts bounded sets; that is, for every bounded set $B$ in $E^{\prime}$, we can find a bounded set $C$ in $\Lambda^{\prime}$ such that $B \subset T(C)$. Hence $U: E \rightarrow \Lambda$ is a topological isomorphism into its image, which means that $\left(g_{i}\right)_{i}$ is a $\Lambda$-frame for $E$.

The following result is the version in the locally convex context of Propositions 2.2 and 3.4 of [10]. It relates $\Lambda$-Bessel sequences with frames with respect to $\Lambda$ when $\Lambda$ is a barreled sequence space for which the canonical unit vectors $\left(e_{i}\right)_{i}$ form a Schauder basis. Note that, if $\left(g_{i}\right)_{i} \subset E^{\prime}$ is a frame with respect to $\Lambda$, then, by definition, $U(E)$ is complemented in $\Lambda$; this means that the operator $U^{-1}: U(E) \rightarrow E$ can be extended to a continuous linear operator $S: \Lambda \rightarrow E$.

Proposition 2.8. Let $E$ be a barreled and complete $L C S$, and let $\Lambda$ be a barreled sequence space for which the canonical unit vectors $\left(e_{i}\right)_{i}$ form a Schauder basis. If $\left(g_{i}\right)_{i} \subset E^{\prime}$ is $\Lambda$-Bessel for $E$, then the following conditions are equivalent:

(1) $\left(g_{i}\right)_{i} \subset E^{\prime}$ is a frame with respect to $\Lambda$.

(2) There exists a sequence $\left(f_{i}\right)_{i} \subset E$ such that $\sum_{i=1}^{\infty} c_{i} f_{i}$ is convergent for every $\left(c_{i}\right)_{i} \in \Lambda$ and $x=\sum_{i=1}^{\infty} g_{i}(x) f_{i}$ for every $x \in E$.

(3) There exists a $\Lambda^{\prime}$-Bessel sequence $\left(f_{i}\right)_{i} \subset E \subseteq E^{\prime \prime}$ for $E^{\prime}$ such that $x=$ $\sum_{i=1}^{\infty} g_{i}(x) f_{i}$ for every $x \in E$.

If the canonical unit vectors form a basis for both $\Lambda$ and $\Lambda_{\beta}^{\prime}$, then (1)-(3) are also equivalent to the following:

(4) There exists a $\Lambda^{\prime}$-Bessel sequence $\left(f_{i}\right)_{i} \subset E \subseteq E^{\prime \prime}$ for $E^{\prime}$ such that $x^{\prime}=$ $\sum_{i=1}^{\infty} x^{\prime}\left(f_{i}\right) g_{i}$ for every $x^{\prime} \in E^{\prime}$ with convergence in the strong topology.

If each of the cases (3) and (4) hold, then $\left(f_{i}\right)_{i}$ is actually a frame for $E^{\prime}$ with respect to $\Lambda^{\prime}$. Moreover, $\left(\left(g_{i}\right)_{i},\left(f_{i}\right)_{i}\right)$ is a shrinking Schauder frame.

Proof. The equivalence among (1), (2), and (3) can be obtained as in [10].

$(3) \Rightarrow(4)$ If (3) is valid, there exists $\left(f_{i}\right)_{i} \subset E \subseteq E^{\prime \prime}$ that is $\Lambda^{\prime}$-Bessel for $E^{\prime}$ such that $x=\sum_{i=1}^{\infty} g_{i}(x) f_{i}$. Observe that, as $\left(x^{\prime}\left(f_{i}\right)\right)_{i}$ belongs to $\Lambda^{\prime}$, then $\left(x^{\prime}\left(f_{i}\right)\right)_{i}=\sum_{i=1}^{\infty} x^{\prime}\left(f_{i}\right) z_{i}$ is in $\left(\Lambda^{\prime}, \beta\left(\Lambda^{\prime}, \Lambda\right)\right)$, where $\left(z_{i}\right)_{i}$ are the canonical unit vectors of $\Lambda^{\prime}$. Given a bounded set $B \subset E$, then $C=\left\{\left(g_{i}(x)\right): x \in B\right\}$ is a bounded set in $\Lambda$. If $p_{B} \in \operatorname{CS}\left(E_{\beta}^{\prime}\right)$ is the continuous seminorm defined by $p_{B}\left(u^{\prime}\right):=\sup _{x \in B}\left|u^{\prime}(x)\right|$, then

$$
\begin{aligned}
p_{B}\left(x^{\prime}-\sum_{i=1}^{n} x^{\prime}\left(f_{i}\right) g_{i}\right) & =\sup _{x \in B}\left|x^{\prime}(x)-\sum_{i=1}^{n} x^{\prime}\left(f_{i}\right) g_{i}(x)\right| \\
& =\sup _{x \in B}\left|x^{\prime}\left(\sum_{i=1}^{\infty} g_{i}(x) f_{i}\right)-\sum_{i=1}^{n} x^{\prime}\left(f_{i}\right) g_{i}(x)\right| \\
& =\sup _{x \in B}\left|\sum_{i=n+1}^{\infty} x^{\prime}\left(f_{i}\right) g_{i}(x)\right|=\sup _{\phi \in C}\left|\phi\left(\sum_{i=n+1}^{\infty} x^{\prime}\left(f_{i}\right) z_{i}\right)\right| \\
& =q_{C}\left(\sum_{i=n+1}^{\infty} x^{\prime}\left(f_{i}\right) z_{i}\right),
\end{aligned}
$$


where $q_{C} \in \operatorname{CS}\left(\Lambda^{\prime}\right)$ is given by $q_{C}(\alpha):=\sup _{\phi \in C}|\phi(\alpha)|$ for every $\alpha \in \Lambda_{\beta}^{\prime}$. Then $q_{C}\left(\sum_{i=n+1}^{\infty} x^{\prime}\left(f_{i}\right) z_{i}\right)$ converges to 0 as $n$ goes to infinity since $\left(x^{\prime}\left(f_{i}\right)\right)_{i}=$
$\sum_{n=1}^{\infty} x^{\prime}\left(f_{i}\right) z_{i}$ in $\Lambda_{\beta}^{\prime}$.

$(4) \Rightarrow(3)$ If (4) is valid, then there exists $\left(f_{i}\right)_{i}$ a $\Lambda^{\prime}$-Bessel sequence for $E^{\prime}$ such that $x^{\prime}=\sum_{i=1}^{\infty} x^{\prime}\left(f_{i}\right) g_{i}$. Given a bounded subset $B^{\prime} \subset E^{\prime}$, then $C^{\prime}=\left\{\left(x^{\prime}\left(f_{i}\right)\right)\right.$ : $\left.x^{\prime} \in B^{\prime}\right\}$ is a bounded set in $\Lambda_{\beta}^{\prime}$. If $p_{B^{\prime}} \in \mathrm{CS}(E)$ is the continuous seminorm defined by $p_{B^{\prime}}(x):=\sup _{x^{\prime} \in B^{\prime}}\left|x^{\prime}(x)\right|$, then

$$
\begin{aligned}
p_{B^{\prime}}\left(x-\sum_{i=1}^{n} g_{i}(x) f_{i}\right) & =\sup _{x^{\prime} \in B^{\prime}}\left|x^{\prime}(x)-\sum_{i=1}^{n} x^{\prime}\left(f_{i}\right) g_{i}(x)\right| \\
& =\sup _{x^{\prime} \in B^{\prime}}\left|\sum_{i=n+1}^{\infty} x^{\prime}\left(f_{i}\right) g_{i}(x)\right|=\sup _{\phi^{\prime} \in C^{\prime}}\left|\phi^{\prime}\left(\sum_{i=n+1}^{\infty} g_{i}(x) e_{i}\right)\right| \\
& =q\left(\sum_{i=n+1}^{\infty} g_{i}(x) e_{i}\right)
\end{aligned}
$$

where $q$ is a continuous seminorm in $\Lambda_{\beta}$. Then $q\left(\sum_{i=n+1}^{\infty} g_{i}(x) e_{i}\right)$ converges to 0 as $n$ goes to infinity due to the fact that $\left(g_{i}(x)\right)_{i}=\sum_{i=1}^{\infty} g_{i}(x) e_{i}$ in $\Lambda$.

To conclude, observe that, if (3) and (4) hold, then $\left(\left(g_{i}\right)_{i},\left(f_{i}\right)_{i}\right)$ and $\left(\left(f_{i}\right)_{i},\left(g_{i}\right)_{i}\right)$ are Schauder frames for $E$ and $E^{\prime}$, respectively. By [8, Proposition 2.3] we obtain that $\left(\left(g_{i}\right)_{i},\left(f_{i}\right)_{i}\right)$ is a shrinking Schauder frame.

A locally convex algebra is an LCS which is an algebra with separately continuous multiplication. The spectrum of the algebra is the set of all nonzero multiplicative linear functionals. The following remark will be useful in Section 4.2.

Remark 2.9. (1) In many cases $E$ is continuously included in a locally convex algebra $\mathcal{A}$ with nonempty spectrum, $\Lambda$ is a solid sequence space, $\left(g_{i}\right)_{i}$ is a $\Lambda$-frame, and every $g_{i}$ is the restriction to $E$ of a continuous linear multiplicative functional on $\mathcal{A}$. Let us assume that, for some $a \in \mathcal{A}$, the operator

$$
T: E \rightarrow E(\subset \mathcal{A}), \quad x \mapsto a x
$$

is well defined and it is a topological isomorphism into its image, and that $\alpha:=$ $\left(g_{i}(a)\right)_{i}$ defines by pointwise multiplication a continuous operator on $\Lambda$. Then $\left(h_{i}\right)_{i}$, where

$$
h_{i}:= \begin{cases}g_{i} & \text { if } g_{i}(a) \neq 0 \\ 0 & \text { if } g_{i}(a)=0\end{cases}
$$

is a $\Lambda$-frame. In fact, since $U \circ T$ is a topological isomorphism into its image, then, for every continuous seminorm $p$ on $E$, there is a continuous seminorm $q$ on $\Lambda$ such that

$$
p(x) \leq q\left(\left(g_{i}(a x)\right)_{i}\right)=q\left(\left(g_{i}(a) g_{i}(x)\right)_{i}\right)=q\left(\left(g_{i}(a) h_{i}(x)\right)_{i}\right) .
$$

Finally, since the pointwise multiplication with $\left(g_{i}(a)\right)_{i}$ is a continuous operator on $\Lambda$, we find a continuous seminorm $r$ on $\Lambda$ with

$$
p(x) \leq r\left(\left(h_{i}(x)\right)_{i}\right), \quad x \in E .
$$


(2) If $E$ is a locally convex algebra with a nonempty spectrum, $\Lambda$ is a barreled sequence space, and $\left(g_{i}\right)_{i}$ is a $\Lambda$-frame consisting of continuous linear multiplicative functionals on $E$, then $U(E)$ is a locally convex algebra under pointwise multiplication. Hence, if $E$ has no zero-divisors, then the analysis map $U$ cannot be surjective. In fact, if there are $x, y \in E$ such that $U(x)=e_{1}$ and $U(y)=e_{2}$, then

$$
U(x \cdot y)=\left(g_{i}(x \cdot y)\right)_{i}=\left(g_{i}(x) g_{i}(y)\right)_{i}=0
$$

and the injectivity of $U$ implies $x \cdot y=0$, which is a contradiction. Since the range of $U$ is a topological subspace of $\Lambda$, the nonsurjectivity of $U$ implies the noninjectivity of the transpose map $U^{\prime}$. Consequently, the expression of any element in $E^{\prime}$ as a convergent series

$$
\sum_{i} \alpha_{i} g_{i}
$$

with $\alpha \in \Lambda^{\prime}$ is never unique.

\section{3. $\Lambda$-FRAMES FOR $(L B)$-SPACES}

Let $E=\operatorname{ind}_{n}\left(E_{n},\|\cdot\|_{n}\right)$ and $\Lambda=\operatorname{ind}_{n}\left(\Lambda_{n}, r_{n}\right)$ be complete $(L B)$-spaces, and let $\left(g_{i}\right)_{i} \subset E^{\prime}$ be a $\Lambda$-Bessel sequence. Let $U: E \rightarrow \Lambda$ be the continuous and linear map of Definition 2.1, and, for each $n \in \mathbb{N}$, consider the seminormed space $\left(F_{n}, q_{n}\right)$, where

$$
F_{n}=\left\{x \in E: U(x) \in \Lambda_{n}\right\}
$$

and $q_{n}(x):=r_{n}(U(x))$. Let us consider the topologies on $E$,

$$
\left(E, \tau_{1}\right)=\operatorname{ind}_{n}\left(E_{n},\|\cdot\|_{n}\right), \quad\left(E, \tau_{2}\right)=\operatorname{ind}_{n}\left(F_{n}, q_{n}\right) .
$$

Finally, denote by $\tau_{3}$ the topology on $E$ given by the system of seminorms $x \mapsto$ $p(U(x))$ when $p$ runs in $\operatorname{CS}(\Lambda)$. Then

$$
\tau_{1} \geq \tau_{2} \geq \tau_{3}
$$

but observe that $\tau_{2}$ and $\tau_{3}$ need not be even Hausdorff. This notation will be kept through all this section.

We observe that $\left(g_{i}\right)_{i} \subset E^{\prime}$ is a $\Lambda$-frame if and only if the former three topologies coincide.

The coincidence $\tau_{1}=\tau_{2}$ is easily characterized under the mild additional assumption that the closed unit ball of $\Lambda_{n}$ is also closed in $\omega$. This is the case for all (weighted) $\ell_{p}$ spaces, $1 \leq p \leq \infty$, but not for $c_{0}$.

Applications of the results in this section for weakly sufficient sets will be given in Section 4. We refer to [23, Section 8.5] for the behavior of bounded sets and convergent sequences in $(L B)$-spaces.

Proposition 3.1. Assume that the closed unit ball of $\Lambda_{n}$ is closed in $\omega$ for each $n \in \mathbb{N}$. Then $\tau_{1}=\tau_{2}$ if and only if $\left(F_{n}, q_{n}\right)$ is a Banach space for each $n \in \mathbb{N}$.

Proof. Assume that $\tau_{1}=\tau_{2}$, which in particular implies that $\tau_{2}$ is Hausdorff. Since $\left(F_{n}, q_{n}\right)$ is continuously injected in $\left(E, \tau_{2}\right), q_{n}$ is a norm. Moreover, if $x \in E, x \neq 0$, then there is $n$ such that $x \in F_{n}$; hence $q_{n}(x)>0$. We have $0<q_{n}(x)=r_{n}(U(x))$, which implies $U(x) \neq 0$. Thus $U$ is injective. Let $\left(x_{j}\right)_{j}$ be a Cauchy sequence in 
$\left(F_{n}, q_{n}\right)$. Then it converges to a vector $x$ in the complete $(L B)$-space $E$, and therefore its image under the analysis map $\left(U\left(x_{j}\right)\right)_{j}$ is convergent to $U(x)$ in $\Lambda$. Now, given $\varepsilon>0$, we can find $j_{0}$ such that $r_{n}\left(U\left(x_{j}\right)-U\left(x_{k}\right)\right) \leq \varepsilon$ whenever $j, k \geq j_{0}$; that is, for $k \geq j_{0}$,

$$
U\left(x_{k}\right) \in U\left(x_{j}\right)+\left\{\alpha \in \Lambda_{n}: r_{n}(\alpha) \leq \varepsilon\right\},
$$

and then

$$
U(x) \in{\overline{U\left(x_{j}\right)+\left\{\alpha \in \Lambda_{n}: r_{n}(\alpha) \leq \varepsilon\right\}}}^{\omega}
$$

for all $j \geq j_{0}$. By hypothesis, we get $U(x) \in \Lambda_{n}$ and $r_{n}\left(U\left(x-x_{j}\right)\right) \leq \varepsilon$ for all $j \geq k_{0}$. Hence $\left(F_{n}, q_{n}\right)$ is a Banach space.

The converse holds since by the open mapping theorem two comparable $(L B)$ topologies must coincide.

The next result depends on Grothendieck's factorization theorem (see [23, Theorem 1.2.20]).

Corollary 3.2. Assume that the closed unit ball of $\Lambda_{n}$ is closed in $\omega$. Then $\tau_{1}=\tau_{2}$ if and only if for each $n$ there are $m$ and $C$ such that $F_{n} \subset E_{m}$ and

$$
\|x\|_{m} \leq C q_{n}(x)
$$

for each $x \in F_{n}$.

Proposition 3.3. If $E$ is Montel and $\tau_{1}=\tau_{2}$, then $\left(g_{i}\right)_{i}$ is a $\Lambda$-frame for $E$.

Proof. As in Proposition 3.1, $U$ is injective and each $\left(F_{n}, q_{n}\right)$ is a normed space. By Baernstein's lemma (see [23, Theorem 8.3.55]), as $E$ is a Montel space and $\Lambda$ is a complete $(L B)$-space, it suffices to show that, for each bounded subset $B$ of $\Lambda$, the pre-image $U^{-1}(B)$ is bounded in $\left(E, \tau_{2}\right)$. As $\Lambda$ is regular, because it is complete, there is $n$ such that $B$ is contained and bounded in $\Lambda_{n}$; hence $U^{-1}(B)$ is contained and bounded in $F_{n}$, and therefore bounded in $E$.

Our next result is an abstract version of [1, Theorems 2 and 3]. Recall that a $(D F S)$-space is an $(L B)$-space $E=\operatorname{ind}_{n}\left(E_{n},\|\cdot\|_{n}\right)$ such that, for each $n$, there is $m>n$ such that the inclusion map $E_{n} \longleftrightarrow E_{m}$ is compact.

Theorem 3.4. Let $E=\operatorname{ind}_{n}\left(E_{n},\|\cdot\|_{n}\right)$ be a $(D F S)$-space, and let $\Lambda=\operatorname{ind}_{n}\left(\Lambda_{n}\right.$, $\left.r_{n}\right)$ be a complete $(L B)$-space. Assume that the closed unit ball of $\Lambda_{n}$ is closed in $\omega$. If $\left(g_{i}\right)_{i} \subset E^{\prime}$ is a $\Lambda$-Bessel sequence, then the following conditions are equivalent:

(1) $\left(g_{i}\right)_{i}$ is a $\Lambda$-frame,

(2) The map $U: E \rightarrow \Lambda, U(x)=\left(g_{i}(x)\right)_{i}$, is injective, and for every $n \in \mathbb{N}$ there exists $m>n$ such that $F_{n} \subset E_{m}$.

Proof. If (1) is satisfied, then $\tau_{1}=\tau_{2}=\tau_{3}$. The injectivity of $U$ follows as in the proof of Proposition 3.1, and the rest of (2) follows by Corollary 3.2.

We prove that (2) implies (1). Without loss of generality, we can assume that $E_{n} \subset E_{n+1}$ with compact inclusion, $E_{n} \subset F_{n}, q_{n}(x) \leq\|x\|_{n}$, and $\|x\|_{n+1} \leq\|x\|_{n}$ for all $x \in E_{n}$ and for every $n$. It suffices to show that, under condition (2), the inclusion $F_{n} \subset E_{m+2}$ is continuous. In fact, this implies the coincidence of the topologies $\tau_{1}=\tau_{2}$, and hence the $\Lambda$-frame property by Proposition 3.3. 
Fix $n \in \mathbb{N}$ and define

$$
B=\left\{x \in F_{n}: q_{n}(x) \leq 1,\|x\|_{m+1}>1\right\}
$$

and

$$
A=\left\{y=\frac{x}{\|x\|_{m+1}}: x \in B\right\} .
$$

We can assume $B$ is an infinite set since otherwise the inclusion $F_{n} \subset E_{m+1}$ is continuous and we are done. Let $\left(p_{j}\right)_{j}$ denote a fundamental system of seminorms for the Fréchet space $\omega$. We claim that there are $j_{0} \in \mathbb{N}$ and $C>0$ such that

$$
\|x\|_{m+1} \leq C p_{j_{0}}(U(x))
$$

for every $x \in B$. Otherwise there is a sequence $\left(y_{j}\right)_{j} \subset A$ such that

$$
p_{j}\left(U\left(y_{j}\right)\right) \leq \frac{1}{j !} .
$$

Assume that $\left(y_{j}\right)_{j}$ is bounded in $E_{m}$. Then it would be relatively compact in $E_{m+1}$. Therefore, there is a subsequence $\left(y_{s}\right)_{s}$ of $\left(y_{j}\right)_{j}$ that converges to $y$ in $E_{m+1}$. Hence $U\left(y_{s}\right) \rightarrow U(y)$ in $\Lambda$, and hence in $\omega$. We can apply (1) to conclude that $U(y)=0$, and hence $y=0$ since $U$ is injective. This contradicts $\left\|y_{s}\right\|_{m+1}=1$ for all $s$. Consequently, $\left(y_{j}\right)_{j}$ is unbounded in $E_{m}$. Hence, for $j_{1}=1$, there exists $j_{2}>j_{1}$ such that

$$
\frac{1}{6 \cdot 2^{2}}\left\|y_{j_{2}}\right\|_{m}>3\left\|y_{j_{1}}\right\|_{m} .
$$

There is $\psi$ in the unit ball $B_{E_{m}^{\prime}}$ of $E_{m}^{\prime}$ such that

$$
\frac{1}{6 \cdot 2^{2}}\left|\psi\left(y_{j_{2}}\right)\right|>3\left\|y_{j_{1}}\right\|_{m}>2\left|\psi\left(y_{j_{1}}\right)\right| \text {. }
$$

Since $U: E_{m} \rightarrow \omega$ is a continuous and injective map, then $U^{\prime}: \omega^{\prime} \rightarrow E_{m}^{\prime}$ has $\sigma\left(E_{m}^{\prime}, E_{m}\right)$-dense range, and we can find $\varphi_{2} \in \omega^{\prime}$ such that

$$
\max _{k=1,2}\left|\left(\varphi_{2} \circ U-\psi\right)\left(y_{j_{k}}\right)\right|
$$

is so small that

$$
\frac{1}{6 \cdot 2^{2}}\left|\varphi_{2}\left(U\left(y_{j_{2}}\right)\right)\right|>3\left\|y_{j_{1}}\right\|_{m}>2\left|\varphi_{2}\left(U\left(y_{j_{1}}\right)\right)\right| .
$$

By condition (1) there is $j_{2}^{\prime}$ such that

$$
\left|\varphi_{2}\left(U\left(y_{j}\right)\right)\right|<\left|\varphi_{2}\left(U\left(y_{j_{2}}\right)\right)\right|, \quad j>j_{2}^{\prime} .
$$

Proceeding by induction, it is possible to obtain a sequence $\left(\varphi_{\ell}\right)_{\ell} \subset \omega^{\prime}$ and an increasing sequence $\left(j_{\ell}\right)_{\ell}$ of indices such that $\varphi_{\ell} \circ U \in B_{E_{m}^{\prime}}$ and

$$
\frac{1}{\ell(\ell+1) 2^{\ell}}\left|\varphi_{\ell}\left(U\left(y_{j_{\ell}}\right)\right)\right|>3 \sum_{k=1}^{\ell-1}\left\|y_{j_{k}}\right\|_{m}>2 \sum_{k=1}^{\ell-1}\left|\varphi_{\ell}\left(U\left(y_{j_{k}}\right)\right)\right|
$$

while

$$
\left|\varphi_{\ell}\left(U\left(y_{j_{k}}\right)\right)\right|<\left|\varphi_{\ell}\left(U\left(y_{j_{\ell}}\right)\right)\right| \quad \forall k>\ell
$$


We now consider

$$
y=\sum_{k=1}^{\infty} \frac{1}{k 2^{k}} y_{j_{k}} \in E_{m+1} .
$$

Then

$$
\varphi_{\ell}(U(y))=\sum_{k=1}^{\infty} \frac{1}{k 2^{k}} \varphi_{\ell}\left(U\left(y_{j_{k}}\right)\right)
$$

and hence

$$
\begin{aligned}
\left|\varphi_{\ell}(U(y))\right| & \geq \frac{1}{\ell 2^{\ell}}\left|\varphi_{\ell}\left(U\left(y_{j_{\ell}}\right)\right)\right|-\sum_{k<\ell} \frac{1}{k 2^{k}}\left|\varphi_{\ell}\left(U\left(y_{j_{k}}\right)\right)\right|-\sum_{k>\ell}^{\infty} \frac{1}{k 2^{k}}\left|\varphi_{\ell}\left(U\left(y_{j_{k}}\right)\right)\right| \\
& \geq\left(\frac{1}{\ell 2^{\ell}}-\sum_{k>\ell} \frac{1}{k 2^{k}}\right)\left|\varphi_{\ell}\left(U\left(y_{j_{\ell}}\right)\right)\right|-\sum_{k<\ell} \frac{1}{k 2^{k}}\left|\varphi_{\ell}\left(U\left(y_{j_{k}}\right)\right)\right| \\
& \geq \frac{1}{\ell(\ell+1) 2^{\ell}}\left|\varphi_{\ell}\left(U\left(y_{j_{\ell}}\right)\right)\right|-\frac{3}{2} \sum_{k<\ell}\left\|y_{j_{k}}\right\|_{m} \\
& \geq \sum_{k<\ell}\left\|y_{j_{k}}\right\|_{m} \geq \sum_{k<\ell}\left\|y_{j_{k}}\right\|_{m+1}=\ell-1 .
\end{aligned}
$$

On the other hand, $r_{n}\left(U\left(y_{j_{k}}\right)\right) \leq 1$ for every $k \in \mathbb{N}$, which implies that the series

$$
\sum_{k=1}^{\infty} \frac{1}{k 2^{k}} U\left(y_{j_{k}}\right)
$$

converges in the Banach space $\Lambda_{n}$. Hence $U(y)$ belongs to $\Lambda_{n}$, and $y \in F_{n} \subset E_{m}$. Since $\varphi_{\ell} \circ U \in B_{E_{m}^{\prime}}$, then $\left|\varphi_{\ell}(U(y))\right| \leq 1$, which is a contradiction. Consequently, the claim is proved, and there are $j_{0} \in \mathbb{N}$ and $C>0$ such that

$$
\|x\|_{m+1} \leq C p_{j_{0}}(U(x))
$$

for every $x \in B$. In order to conclude that the inclusion $F_{n} \subset E_{m+2}$ is continuous, it suffices to check that $B$ is bounded in $E_{m+2}$. To this end, we first observe that

$$
1 \leq\|x\|_{m+1} \leq C p_{j_{0}}(U(x)) \leq C^{\prime}\|x\|_{m+2}
$$

for some $C^{\prime}>0$ and for all $x \in B$. Then

$$
\left\{\frac{x}{\|x\|_{m+2}}: x \in B\right\} \subset E_{m}
$$

is a bounded set in $E_{m+1}$, and hence relatively compact in $E_{m+2}$. We now proceed by contradiction and assume that $B$ is unbounded in $E_{m+2}$. Then there exists a sequence $\left(x_{j}\right)_{j} \subset B$ with $\left\|x_{j}\right\|_{m+2} \geq j$. Passing to a subsequence if necessary, we can assume that

$$
z_{j}:=\frac{x_{j}}{\left\|x_{j}\right\|_{m+2}}
$$

converges to some element $z \in E_{m+2}$ such that $\|z\|_{m+2}=1$. Since the inclusion $E_{m+2} \subset F_{m+2}$ is continuous, we get

$$
\lim _{j \rightarrow \infty} q_{m+2}\left(z_{j}-z\right)=0
$$


From the injectivity of $U$ we get $q_{m+2}(z)=r_{m+2}(U(z))=a>0$, and there is $j_{1} \in \mathbb{N}$ such that $q_{m+2}\left(z_{j}\right) \geq \frac{a}{2}$ whenever $j \geq j_{1}$, which implies $q_{m+2}\left(x_{j}\right) \geq \frac{a}{2} j$ for all $j \geq j_{1}$. This is a contradiction since $(m>n)$

$$
q_{m+2}\left(x_{j}\right) \leq q_{n}\left(x_{j}\right) \leq 1 .
$$

The proof is complete.

\section{EXAmples}

4.1. Weighted spaces of holomorphic functions. Let $G$ be either an open disc centered at the origin or $\mathbb{C}$. A radial weight on $G$ is a strictly positive continuous function $v$ on $G$ such that $v(z)=v(|z|), z \in G$. Then the weighted Banach space of holomorphic functions is defined by

$$
H v(G):=\left\{f \in \mathcal{H}(G):\|f\|_{v}:=\sup _{z \in G} v(|z|)|f(z)|<+\infty\right\} .
$$

The closed subspace of $H v(G)$ consisting of those $f \in \mathcal{H}(G)$ such that $v f$ vanishes at infinity on $G$ is denoted $H(v)_{0}(G)$.

Let $V=\left(v_{n}\right)_{n}$ be a decreasing sequence of weights on $G$. Then the weighted inductive limit of spaces of holomorphic functions is defined by

$$
V H(G):=\operatorname{ind}_{n} H v_{n}(G)
$$

that is, $V H(G)$ is the increasing union of the Banach spaces $H v_{n}(G)$ endowed with the strongest locally convex topology for which all the injections $H v_{n}(G) \rightarrow$ $V H(G)$ become continuous.

Similarly, given an increasing sequence of weights $W=\left(w_{n}\right)_{n}$ on $G$, the weighted projective limit of spaces of entire functions is defined by

$$
H W(G):=\operatorname{proj}_{n} H w_{n}(G) ;
$$

that is, $H W(G)$ is the decreasing intersection of the Banach spaces $H w_{n}(G)$ whose topology is defined by the sequence of norms $\|\cdot\|_{w_{n}}$. It is a Fréchet space.

In both cases, when $G=\mathbb{C}$ we will simply write $V H$ and $H W$.

Given any sequence $S:=\left(z_{i}\right)_{i} \subset G$ and a decreasing sequence of weights $V$ on $G$, put

$$
\nu_{n}(i)=v_{n}\left(z_{i}\right)
$$

and

$$
V \ell_{\infty}(S)=\operatorname{ind}_{n} \ell_{\infty}\left(\nu_{n}\right)
$$

For an increasing sequence of weights $W=\left(w_{n}\right)_{n}$ on $G$, put

$$
\omega_{n}(i):=w_{n}\left(z_{i}\right)
$$

and

$$
\ell_{\infty} W(S)=\bigcap_{n} \ell_{\infty}\left(\omega_{n}\right)
$$

Obviously, the restriction maps

$$
R: V H(G) \rightarrow V \ell_{\infty}(S), \quad f \mapsto\left(f\left(z_{i}\right)\right)_{i}
$$


and

$$
R: H W(G) \rightarrow \ell_{\infty} W(S), \quad f \mapsto\left(f\left(z_{i}\right)\right)_{i}
$$

are well defined and continuous; that is, $\left(\delta_{z_{i}}\right)_{i}$ is a $V \ell_{\infty}(S)$-Bessel sequence for $V H(G)$ and a $\ell_{\infty} W(S)$-Bessel sequence for $H W(G)$. As usual, $\delta_{z}$ denotes the point evaluation at $z$. We want to analyze when these Bessel sequences are in fact frames, that is, when the restriction map is an isomorphism into its image.

Let us first concentrate on the Fréchet case. Then $\left(\delta_{z_{i}}\right)_{i}$ is a $\ell_{\infty} W(S)$-frame if and only if for every $n$ there are $m$ and $C$ such that

$$
\sup _{z \in G}|f(z)| w_{n}(z) \leq C \sup _{i}\left|f\left(z_{i}\right)\right| w_{m}\left(z_{i}\right)
$$

for every $f \in H W(G)$. This is the same as saying that $S$ is a sufficient set for $H W(G)$. The concept of sufficient set was introduced by Ehrenpreis in [11, pp. 3, 4, 13] (see Corollary 4.6 or Proposition 4.7 for some examples).

The $(L B)$-case is more delicate. Following the notation of Section 3, if $E_{n}:=$ $H v_{n}(G)$, then the space $F_{n}:=\left\{f \in V H(G): R(f) \in \ell_{\infty}\left(\nu_{n}\right)\right\}$ is usually denoted by $A\left(S, v_{n}\right)$ and the corresponding seminorm $q_{n}$ is denoted $\|\cdot\|_{n, S}$; that is,

$$
\|f\|_{n, S}=\sup _{i \in \mathbb{N}}\left|f\left(z_{i}\right)\right| \nu_{n}(i), \quad f \in A\left(S, v_{n}\right) .
$$

Then $\tau_{1}$ is the topology of the inductive limit $V H(G)$, and $\tau_{2}$ is the one of $\operatorname{ind}_{n} A\left(S, v_{n}\right)$. We recall that $S$ is said to be weakly sufficient for $V H(G)$ when $V H(G)=\operatorname{ind}_{n} A\left(S, v_{n}\right)$ topologically; that is, $\tau_{1}=\tau_{2}$. It should be mentioned that this definition a priori is not restricted to discrete sets, but this is the most interesting case. We obtain the following general results which have been formulated in one way or another in concrete situations (see in particular [1], [16], [17], [21], [22]).

Theorem 4.1 (see [1, Theorems 2, 3]). The following statements are equivalent:

(1) $S:=\left(z_{i}\right)_{i}$ is weakly sufficient,

(2) $A\left(v_{n}, S\right)$ is a Banach space for every $n \in \mathbb{N}$,

(3) for each $n$ there are $m \geq n$ and $C>0$ such that, for every $f \in V H(G)$, one has

$$
\|f\|_{m} \leq C\|f\|_{n, S}
$$

Proof. Apply Proposition 3.1 and Corollary 3.2.

Theorem 4.2 (see [1, Theorems 2, 3]). Let us assume that $\frac{v_{n+1}}{v_{n}}$ vanishes at infinity on $G$ for every $n \in \mathbb{N}$. Then the following conditions are equivalent:

(1) $S:=\left(z_{i}\right)_{i}$ is weakly sufficient.

(2) The restriction map $V H(G) \rightarrow V \ell_{\infty}(S)$ is injective, and for each $n$ there are $m \geq n$ and $C>0$ such that $A\left(v_{n}, S\right) \subset H v_{m}(G)$.

Proof. The hypothesis on the sequence $\left(v_{n}\right)_{n}$ implies that $V H(G)$ is a $(D F S)$ space (see [6, Theorem 1.6]). Now it suffices to apply Theorem 3.4.

The injectivity of the restriction map means that $S$ is a uniqueness set for $V H(G)$. As a consequence of Proposition 3.3, we obtain the following theorem. 
Theorem 4.3. If $V H(G)$ is Montel, $S$ is weakly sufficient if and only if the restriction map

$$
R: V H(G) \rightarrow V \ell_{\infty}(S),\left.\quad f \mapsto f\right|_{S},
$$

is a topological isomorphism into its image.

Theorem 4.3 asserts that, if the space $V H(G)$ is Montel, the set of Dirac evaluations $\left\{\delta_{z} \mid z \in S\right\} \subset V H(G)^{\prime}$ is a $V \ell_{\infty}(S)$-frame if and only if $S$ is weakly sufficient.

If the sequence $V$ reduces to one weight, then $V=(v),\left(\delta_{z_{i}}\right)_{i}$ is a $\ell_{\infty}(\nu)$-frame for $H v(G)$ if and only if $S$ is a sampling set for $H v(G)$. If $\left(v_{n}\right)_{n}$ is a decreasing sequence of weights on $G$ and $S$ is a sampling set for $H v_{n}(G)$ for each $n$, then $S$ is a weakly sufficient set for $V H(G)$. However, Khoi and Thomas [14] gave examples of countable weakly sufficient sets $S=\left(z_{i}\right)_{i}$ in the space

$$
A^{-\infty}(\mathbb{D}):=\operatorname{ind}_{n} H v_{n}(\mathbb{D}) \quad \text { with } v_{n}(z)=(1-|z|)^{n},
$$

which are not sampling sets for any $H v_{n}(\mathbb{D}), n \in \mathbb{N}$. As $A^{-\infty}(\mathbb{D})$ is Montel, $\left(\delta_{z_{i}}\right)_{i}$ is a $V \ell_{\infty}(S)$-frame for $A^{-\infty}(\mathbb{D})$ which is not a $\ell_{\infty}\left(\nu_{n}\right)$-frame for $H v_{n}(\mathbb{D})$ for any $n$. Bonet and Domanski [7] studied weakly sufficient sets in $A^{-\infty}(\mathbb{D})$ and their relation to what they called $(p, q)$-sampling sets.

The dual of the space $A^{-\infty}(\mathbb{D})$ can be identified via the Laplace transform with the space of entire functions $A_{\mathbb{D}}^{-\infty}:=H W(\mathbb{C})$ for the sequence of weights $W=\left(w_{n}\right)_{n}$,

$$
w_{n}(z)=(1+|z|)^{n} e^{-|z|}
$$

(see [20] and also [2] for the several variables case). In [2] explicit constructions of sufficient sets for this space are given. For instance, for each $k$ take $\ell_{k} \in \mathbb{N}, \ell_{k}>$ $2 \pi k^{2}$, and let $z_{k, j}:=k r_{k, j}, 1 \leq j \leq \ell_{k}$, where $r_{k, j}$ are the $\ell_{k}$-roots of the unity. Then, with an appropriate order, $\left(\delta_{z_{k, j}}: k \in \mathbb{N}, 1 \leq j \leq \ell_{k}\right)$ is a $\ell_{\infty} W(S)$-frame for $A_{\mathbb{D}}^{-\infty}$. More examples for nonradial weights can be found in [3].

Finally, from Proposition 2.6, we recover the following consequence about representing systems. It should be compared with Corollary 4.10 below.

Theorem 4.4 (see [2, Proposition 4.2]). $\quad$ (1) $\left(\lambda_{k}\right)_{k} \subset \mathbb{C}$ is sufficient for $A_{\mathbb{D}}^{-\infty}$ if and only if every function $f \in A^{-\infty}(\mathbb{D})$ can be represented as

$$
f(z)=\sum_{k} \alpha_{k} e^{\lambda_{k} z}
$$

where

$$
\sum_{k}\left|\alpha_{k}\right|\left(1+\left|\lambda_{k}\right|\right)^{-n} e^{\left|\lambda_{k}\right|}<\infty \quad \text { for some } n \in \mathbb{N} .
$$

(2) $\left(\lambda_{k}\right)_{k} \subset \mathbb{D}$ is weakly sufficient in $A^{-\infty}(\mathbb{D})$ if and only if each function $f \in A_{\mathbb{D}}^{-\infty}$ can be represented as

$$
f(z)=\sum_{k} \alpha_{k} e^{\lambda_{k} z},
$$


where

$$
\sum_{k}\left|\alpha_{k}\right|\left(1-\left|\lambda_{k}\right|\right)^{-n}<\infty \quad \text { for every } n \in \mathbb{N} \text {. }
$$

4.2. The Hörmander algebras. In this section we use Landau's notation of little $o$-growth and capital $O$-growth. A function $p: \mathbb{C} \rightarrow[0, \infty[$ is called a growth condition if it is continuous, subharmonic, radial, increases with $|z|$, and satisfies

$(\alpha) \log \left(1+|z|^{2}\right)=o(p(|z|))$ as $|z| \rightarrow \infty$,

( $\beta) p(2|z|)=O(p(|z|))$ as $|z| \rightarrow \infty$.

Given a growth condition $p$, consider the weight $v(z)=e^{-p(|z|)}, z \in \mathbb{C}$, and the decreasing sequence of weights $V=\left(v_{n}\right)_{n}, v_{n}=v^{n}$. We define the following weighted spaces of entire functions (see, e.g., [5]):

$$
A_{p}:=\left\{f \in \mathcal{H}(\mathbb{C}): \text { there is } A>0: \sup _{z \in \mathbb{C}}|f(z)| \exp (-A p(z))<\infty\right\}
$$

that is, $A_{p}=V H$, endowed with the inductive limit topology for which it is a $(D F N)$-algebra (see, e.g., [18]). Given any sequence $S=\left(z_{i}\right)_{i} \subset \mathbb{C}$, we will denote $A_{p}(S)=V \ell_{\infty}(S)$; that is,

$$
A_{p}(S)=\bigcup_{n} \ell_{\infty}\left(\nu_{n}\right), \quad \nu_{n}(i)=e^{-n p\left(\left|z_{i}\right|\right)} .
$$

If we consider the increasing sequence of weights $W=\left(w_{n}\right)_{n}, w_{n}=v^{1 / n}$, then we define

$$
A_{p}^{0}:=\left\{f \in \mathcal{H}(\mathbb{C}): \text { for all } \varepsilon>0: \sup _{z \in \mathbb{C}}|f(z)| \exp (-\varepsilon p(z))<\infty\right\}
$$

that is, $A_{p}^{0}=H W$, endowed with the projective limit topology for which it is a nuclear Fréchet algebra (see, e.g., [19]). Clearly, $A_{p}^{0} \subset A_{p}$. As before, given a sequence $S=\left(z_{i}\right)_{i} \subset \mathbb{C}$, we will denote $A_{p}^{0}(S)=\ell_{\infty} W(S)$; that is,

$$
A_{p}^{0}(S)=\bigcap_{n} \ell_{\infty}\left(\omega_{n}\right), \quad \omega_{n}(i)=e^{-\frac{1}{n} p\left(\left|z_{i}\right|\right)} .
$$

Condition $(\alpha)$ implies that, for each $a>0$, the weight $v_{a}(z):=e^{-a p(|z|)}$ is rapidly decreasing, and, consequently, the polynomials are contained and dense in $H\left(v_{a}\right)_{0}$, and that for $a<b$ the inclusion $H v_{a} \subset H\left(v_{b}\right)_{0}$ is compact. Therefore, the polynomials are dense in $A_{p}$ and in $A_{p}^{0}$. Condition $(\beta)$ implies that both spaces are stable under differentiation.

Weighted algebras of entire functions of this type, usually known as Hörmander algebras, have been considered since the work of Berenstein and Taylor by many authors (see, e.g., [5] and the references therein).

As an example, when $p_{a}(z)=|z|^{a}$, then $A_{p_{a}}$ consists of all entire functions of order $a$ and finite type or order less than $a$, and $A_{p_{a}}^{0}$ is the space of all entire functions of order at most $a$ and type 0 . For $a=1, A_{p_{1}}$ is the space of all entire functions of exponential type, also denoted $\operatorname{Exp}(\mathbb{C})$, and $A_{p_{1}}^{0}$ is the space of entire functions of infraexponential type. 
As it is well known, the Fourier-Borel transform $\mathcal{F}: H(\mathbb{C})^{\prime} \rightarrow \operatorname{Exp}(\mathbb{C})$ defined by $\mathcal{F}(\mu):=\widehat{\mu}$, where $\widehat{\mu}(z):=\mu_{\omega}\left(e^{z \omega}\right)$, is a topological isomorphism. As a consequence, the dual space of $\operatorname{Exp}(\mathbb{C})$ can be identified with the space of entire functions, $H(\mathbb{C})$. In the same way, for $a>1$ and $b$ its conjugate exponent $\left(a^{-1}+b^{-1}=1\right)$ via the Fourier-Borel transform $\mathcal{F}$, we have the following identifications [28]:

$$
\left(A_{p_{a}}\right)^{\prime}=A_{p_{b}}^{0} \quad \text { and } \quad\left(A_{p_{a}}^{0}\right)^{\prime}=A_{p_{b}} .
$$

From every (weakly) sufficient set $\left(z_{j}\right)_{j}$ for $\left(A_{p}\right) A_{p}^{0}$, we can remove finitely many points $\left(z_{j}\right)_{j=1}^{N}$ and still we have a (weakly) sufficient set (see [3, Corollary to Proposition 4]). In fact, take $Q$ as a nonconstant polynomial which vanishes precisely at points $\left(z_{j}\right)_{j=1}^{N}$. Since the multiplication operator

$$
T_{Q}(f)(z)=Q(z) f(z)
$$

is a topological isomorphism from $A_{p}$ (resp., $A_{p}^{0}$ ) into itself and pointwise multiplication by $\left(Q\left(z_{j}\right)\right)_{j}$ is continuous on $A_{p}(S)$ (resp., $\left.A_{p}^{0}(S)\right)$, it suffices to apply Remark 2.9(1).

Now, we give examples of frames of type $\left(\delta_{z_{i}}\right)_{i}$ in these algebras. We deal first with the Fréchet case.

Theorem 4.5. Given a growth condition $q$, let $S:=\left(z_{n}\right)_{n}$ be a sequence in $\mathbb{C}$ with $\lim _{j}\left|z_{j}\right|=\infty$, and assume that there is $C>0$ such that the distance $d(z, S)$ satisfies $d(z, S) \leq C|z| / \sqrt{q(|z|)}$ for all $z \in \mathbb{C}$. Then the sequence $\left(\delta_{z_{j}}\right)_{j}$ is a $A_{p}^{0}(S)$-frame for $A_{p}^{0}$ whenever $p(r)=o(q(r))$ as $r \rightarrow \infty$.

Proof. We take $V(r)=q(r)$. The family $\{a p, a>0\}$ satisfies (i), (ii), and (iii) in [26, p. 178], and the conclusion follows after applying [26, Theorem 5.1].

In particular, if $p(r)=o\left(r^{2}\right)$ as $r \rightarrow \infty$, then we may take $q(r)=r^{2}$.

Corollary 4.6. If $p(r)=o\left(r^{2}\right)$, then for arbitrary $\alpha, \beta>0$ the regular lattice $\{\alpha n+i \beta m: n, m \in \mathbb{Z}\}$ is a sufficient set for $A_{p}^{0}(\mathbb{C})$. In other words, if $S=\left(z_{n, m}\right)$ with $z_{n, m}:=\alpha n+i \beta m$, then the sequence $\left(\delta_{z_{n, m}}\right)$ is a $A_{p}^{0}(S)$-frame for $A_{p}^{0}(\mathbb{C})$.

The former result is also true in the limit case $p(r)=r^{2}$. In fact, we have the following.

Proposition 4.7. If $p(r)=r^{2}$, then for arbitrary $\alpha, \beta>0$ the regular lattice $\{\alpha n+i \beta m: n, m \in \mathbb{Z}\}$ is a sufficient set for $A_{p}^{0}(\mathbb{C})$. In other words, if $S=\left(z_{n, m}\right)$ with $z_{n, m}:=\alpha n+i \beta m$, then the sequence $\left(\delta_{z_{n, m}}\right)$ is a $A_{p}^{0}(S)$-frame for $A_{p}^{0}(\mathbb{C})$.

Proof. First, we observe that, in this case, $A_{p}^{0}(\mathbb{C})$ coincides algebraically and topologically with the intersection

$$
\bigcap_{\gamma>0} \mathcal{F}_{\gamma}^{2}
$$

of the Bargmann-Fock spaces

$$
\mathcal{F}_{\gamma}^{2}:=\left\{h \in H(\mathbb{C}):\|f\|_{\gamma}:=\int_{\mathbb{C}}|f(z)|^{2} e^{-\gamma|z|^{2}} d z<\infty\right\}
$$


Then, by [27], there is $\gamma_{0}$ such that, for $\gamma \geq \gamma_{0}$, we find constants $A_{\gamma}, B_{\gamma}$ such that

$$
A_{\gamma}\|f\|_{\gamma}^{2} \leq \sum_{n, m}\left|f\left(z_{n, m}\right)\right|^{2} e^{-\gamma\left|z_{n, m}\right|^{2}} \leq B_{\gamma}\|f\|_{\gamma}^{2}
$$

To finish, it is enough to observe that in the definition of $A_{p}^{0}(S)$ one can replace the $\ell_{\infty}$-norms by $\ell_{2}$-norms.

According to [26] (see the comments after Corollary 4.9), there is an entire function of order 2 and finite type which vanishes at the lattice points $S=$ $\{n+i m: n, m \in \mathbb{Z}\}$. In the case $r^{2}=o(p(r))$, we have $f \in A_{p}^{0}$, and the restriction map defined on $A_{p}^{0}$ by $\left.f \mapsto f\right|_{S}$ is not injective. Consequently, the lattice points are not a sufficient set for $A_{p}^{0}$. Similarly, the lattice points are not a weakly sufficient set for $A_{p}$ in the case $r^{2}=O(p(r))$.

From [26, Proposition 8.1] and Theorem 4.2 we get the following.

Proposition 4.8. If $p(r)=o\left(r^{2}\right)$, then for arbitrary $\alpha, \beta>0$ the regular lattice $\{\alpha n+i \beta m: n, m \in \mathbb{Z}\}$ is a weakly sufficient set for $A_{p}(\mathbb{C})$. In other words, if $S=\left(z_{n, m}\right)$ where $z_{n, m}:=\alpha n+i \beta m$, then the sequence $\left(\delta_{z_{n, m}}\right)$ is a $A_{p}(S)$-frame for $A_{p}(\mathbb{C})$.

In particular, for the space $\operatorname{Exp}(\mathbb{C})$, the sequence $\left(\delta_{n+i m}\right)_{n, m \in \mathbb{Z}}$ is a $A_{p}(S)$-frame $\left[29\right.$, Theorem 1]. Here $p(z)=|z|$ and $S=(n+i m)_{n, m \in \mathbb{Z}}$.

By Proposition 2.6, if $S=\left(z_{i}\right)_{i} \subset G$ is a discrete (weakly) sufficient set in $H W(G)$ (resp., in $V H(G)$ ), then each element in the dual space can be represented as a convergent series of type

$$
\sum_{i} \alpha_{i} \delta_{z_{i}}
$$

with coefficients in a given sequence space. Since the spaces under consideration are algebras, this representation is not unique by Remark 2.9(2). As in many cases, the dual space can be identified with a weighted space of holomorphic functions (via the Laplace or the Fourier-Borel transform) in such a way that point evaluations $\delta_{z_{i}}$ are identified with the exponentials $e^{z_{i} z}$, and therefore we get a representation of the elements in the dual space as Dirichlet series, thus obtaining as a consequence several known results, for instance, the following.

Corollary 4.9 (see [29, Corollary, p. 208]). Every entire function $f(z)$ can be represented in the form

$$
f(z)=\sum_{n, m=-\infty}^{\infty} a_{n, m} e^{(n+i m) z}
$$

where $\left|a_{n, m}\right| e^{k\left(n^{2}+m^{2}\right)^{1 / 2}} \rightarrow 0$ as $n^{2}+m^{2} \rightarrow+\infty$ for every $k>0$. Such expansion of $f$ is never unique. 
Corollary 4.10. For $a \geq 2$, every function $f \in A_{p_{a}}$ can be represented in the form

$$
f(z)=\sum_{n, m=-\infty}^{\infty} a_{n, m} e^{(n+i m) z}
$$

with coefficients $\left(a_{n, m}\right)$ satisfying

$$
\left|a_{n, m}\right| \leq C \exp \left(-\varepsilon\left(n^{2}+m^{2}\right)^{b / 2}\right)
$$

(b the conjugate of a) for some constants $\varepsilon, C>0$.

Proof. According to Corollary 4.6 and Proposition 4.7, we can see that the sequence $S=\left\{e^{(n+i m) z}: n, m \in \mathbb{Z}\right\} \subset A_{p_{a}}$ is a $A_{p_{b}}^{0}(S)$-frame for $A_{p_{b}}^{0}$. Since the dual space of $\Lambda=A_{p_{b}}^{0}(S)$ is

$$
\Lambda^{\prime}=\left\{\left(a_{n, m}\right):\left|a_{n, m}\right| \exp \left(\varepsilon\left(n^{2}+m^{2}\right)^{b / 2}\right)<\infty \text { for some } \varepsilon>0\right\},
$$

it suffices to apply Proposition 2.6 to conclude.

Acknowledgment. All the authors' work was supported in part by the projects MTM2013-43540-P, ACOMP/2015/186, and GVA Prometeo II/2013/013 (Spain).

\section{REFERENCES}

1. A. V. Abanin, Certain criteria for weak sufficiency (in Russian), Met. Zametki 40 (1986), no. 4, 442-454; English translation in Math. Notes 40 (1986), 757-764. Zbl 0625.46030. MR0873473. 2, 10, 14

2. A. V. Abanin and L. H. Khoi, Dual of the function algebra $A^{-\infty}(D)$ and representation of functions in Dirichlet series, Proc. Amer. Math. Soc. 138 (2010), no. 10, 3623-3635. Zbl 1205.32004. MR2661561. DOI 10.1090/S0002-9939-10-10383-9. 2, 6, 15

3. A. V. Abanin and V. A. Varziev, Sufficient sets in weighted Fréchet spaces of entire functions, Sib. Math. J. 54 (2013), no. 4, 575-587. Zbl 1290.46016. MR3137144. 2, 15, 17

4. T. I. Abanina, On the problem of a frame concept, Nauchnoe obozrenie 9 (2013), 101-104. 4

5. C. A. Berenstein and R. Gay, Complex Analysis and Special Topics in Harmonic Analysis, Springer, New York, 1995. Zbl 0837.30001. MR1344448. DOI 10.1007/978-1-4613-8445-8. 16

6. K. D. Bierstedt, R. Meise, and W. H. Summers, A projective description of weighted inductive limits, Trans. Amer. Math. Soc. 272 (1982), no. 1, 107-160. Zbl 0599.46026. MR0656483. DOI 10.2307/1998953. 14

7. J. Bonet and P. Domański, Sampling sets and sufficient sets for $A^{-\infty}$, J. Math. Anal. Appl. 277 (2003), no. 2, 651-669. Zbl 1019.30026. MR1961252. DOI 10.1016/ S0022-247X(02)00616-9. 2, 15

8. J. Bonet, C. Fernández, A. Galbis, and J. M. Ribera, Shrinking and boundedly complete Schauder frames in Fréchet spaces, J. Math. Anal. Appl. 410 (2014), no. 2, 953-966. Zbl 1307.42027. MR3111881. DOI 10.1016/j.jmaa.2013.09.010. 1, 4, 5, 8

9. D. Carando, S. Lassalle, and P. Schmidberg, The reconstruction formula for Banach frames and duality, J. Approx. Theory 163 (2011), no. 5, 640-651. Zbl 1222.42030. MR2784516. DOI 10.1016/j.jat.2011.02.007. 2

10. P. Casazza, O. Christensen, and D. Stoeva, Frame expansions in separable Banach spaces, J. Math. Anal. Appl, 307 (2005), no. 2, 710-723. Zbl 1091.46007. MR2142455. DOI 10.1016/j.jmaa.2005.02.015. 1, 2, 3, 6, 7

11. L. Ehrenpreis, Fourier Analysis in Several Complex Variables, Pure Appl. Math. 17, Wiley, New York, 1970. Zbl 0195.10401. MR0285849. 14

12. H. Jarchow, Locally Convex Spaces, B. G. Teubner, Stuttgart, 1981. Zbl 0466.46001. MR0632257. 2, 6 
13. V. M. Kadets and Y. F. Korobeinik, Representing and absolutely representing systems, Studia Math. 102 (1992), no. 3, 217-223. Zbl 0811.46005. MR1170552. 1, 5

14. L. H. Khoi and P. J. Thomas, Weakly sufficient sets for $A^{-\infty}(D)$, Publ. Mat. 42 (1998), no. 2, 435-448. Zbl 1140.46311. MR1676037. DOI 10.5565/PUBLMAT_42298_10. 2, 15

15. Y. F. Korobeinik, Representing systems (in Russian), Uspekhi Mat. Nauk. 36 (1981), no. 1, 73-126; English translation in Russian Math. Surveys 36 (1981), 75-137. Zbl 0483.30003. MR0608942. 5

16. Y. F. Korobeinik, Inductive and projective topologies: Sufficient sets (in Russian), Dokl. Akad. Nauk SSSR 288 (1986), no. 3, 535-539; English translation in Soviet Math. Dokl. 33 (1986), no. 3, 704-707. Zbl 0651.46033. MR0847783. 14

17. Y. F. Korobeinik, Inductive and projective topologies: Sufficient sets and representing systems (in Russian), Izv. Akad. Nauk SSSR 50 (1986), no. 3, 539-565; English translation in Math. USSR-Izv. 28 (1987), no. 3, 529-554. Zbl 0628.46004. MR0854595. 2, 14

18. R. Meise, Sequence space representations for $(D F N)$-algebras of entire functions modulo closed ideals, J. Reine Angew. Math. 363 (1985), 59-95. Zbl 0574.46043. MR0814015. DOI 10.1515/crll.1985.363.59. 16

19. R. Meise and B. A. Taylor, Sequence space representations for (FN)-algebras of entire functions modulo closed ideals, Studia Math. 85 (1987), no. 3, 203-227. Zbl 0669.46002. MR0887485. 16

20. S. N. Melikhov, (DFS)-spaces of holomorphic functions invariant under differentiation, J. Math. Anal. Appl. 297 (2004), no. 2, 577-586. Zbl 1068.46017. MR2088681. DOI 10.1016/j.jmaa.2004.03.030. 2, 15

21. V. V. Napalkov, Comparison of topologies in some spaces of entire functions (in Russian), Dokl. Akad. Nauk SSSR 264 (1982), no. 4, 827-830; English translation in Soviet Math. Dokl. 25 (1982), no. 3, 755-758. Zbl 0551.32003. MR0659583. 2, 14

22. V. V. Napalkov, Strict topology in certain weighted spaces of functions, Math. Notes 39 (1986), no. 4, 291-296. Zbl 0615.46026. MR0842883. 14

23. P. Pérez and J. Bonet, Barrelled Locally Convex Spaces, North-Holland Math. Stud. 131, North-Holland, Amsterdam, 1987. Zbl 0614.46001. MR0880207. 2, 9, 10

24. S. Pilipovic and D. Stoeva, Series expansions in Fréchet spaces and their duals, construction of Fréchet frames, J. Approx. Theory 163 (2011), no. 11, 1729-1747. Zbl 1231.42030. MR2832727. DOI 10.1016/j.jat.2011.06.010. 2

25. S. Pilipovic and D. Stoeva, Fréchet frames, general definition and expansions, Anal. Appl. 12 (2014), no. 2, 195-208. Zbl 1290.42058. MR3189561. DOI 10.1142/S0219530514500018. 2

26. D. M. Schneider, Sufficient sets for some spaces of entire functions, Trans. Amer. Math. Soc. 197 (1974), 161-180. Zbl 0264.46018. MR0357835. 2, 17, 18

27. K. Seip and R. Wallstén, Density theorems for sampling and interpolation in the BargmannFock space II, J. Reine Angew. Math. 429 (1992), 107-113. Zbl 0745.46033. MR1173118. 18

28. B. A. Taylor, Some locally convex spaces of entire functions, Proc. Sympos. Pure Math. 11 (1968), 431-467. Zbl 0181.13304. 17

29. B. A. Taylor, Discrete sufficient sets for some spaces of entire functions, Trans. Amer. Math. Soc. 163 (1972), 207-214. Zbl 0231.46050. MR0290084. 2, 18

\footnotetext{
${ }^{1}$ Instituto Universitario de Matemática Pura y Aplicada IUMPA, Universitat Politècnica de València, E-46071 València, Spain.

E-mail address: jbonet@mat.upv.es; juaripuc@mat.upv.es

${ }^{2}$ Departament D'AnÀlisi Matemàtica, Universitat de VAlÈncia, E-46100 BurJasSOT (VALÈnCIA), SPAIN.

E-mail address: fernand@uv.es; antonio.galbis@uv.es
} 